\title{
Positive solutions to second-order singular nonlocal problems: existence and sharp conditions
}

Shiqi Ma ${ }^{1}$ and Xuemei Zhang ${ }^{1 *}$

"Correspondence: zxm74@sina.com 'School of Mathematics and Physics, North China Electric Power University, Beijing, People's Republic of China

\section{Springer}

\begin{abstract}
In this paper we consider sharp conditions on $\omega$ and $f$ for the existence of $C^{1}[0,1]$ positive solutions to a second-order singular nonlocal problem $u^{\prime \prime}(t)+\omega(t) f(t, u(t))=0$, $u(0)=u(1)=\int_{0}^{1} g(t) u(t) d t$; it turns out that this case is more difficult to handle than two point boundary value problems and needs some new ingredients in the arguments. On the technical level, we adopt the topological degree method.
\end{abstract}

Keywords: Sharp conditions; Singular boundary value problems with integral boundary conditions; Hölder's inequality; Fixed point theorems; Positive solutions

\section{Introduction}

We consider sharp conditions for the second-order singular differential equation with integral boundary conditions

$$
\left\{\begin{array}{l}
u^{\prime \prime}(t)+\omega(t) f(t, u(t))=0, \quad t \in J, \\
u(0)=u(1)=\int_{0}^{1} g(t) u(t) d t
\end{array}\right.
$$

where $J=(0,1), \omega$ is $L^{p}$-integrable on $[0,1]$ for some $1 \leq p \leq+\infty, f$ may be singular at $t=0$ and/or 1 .

In addition, $\omega$ and $f$ satisfy the following conditions:

$\left(H_{1}\right) \omega \in L^{p}[0,1]$ and there exists $\zeta>0$ such that $\omega(t) \geq \zeta$ a.e. on $J$;

$\left(H_{2}\right) f(t, u): J \times[0,+\infty) \rightarrow[0,+\infty)$ is continuous;

$\left(H_{3}\right) g \in L^{1}[0,1]$ is positive with $\mu \in[0,1)$, where

$$
\mu=\int_{0}^{1} g(t) d t
$$

The theory of boundary value problems with positive solutions originates from various real life problems, such as plasma physics, gas dynamics, and chemical reaction. The study of boundary value problems with positive solutions has attracted recently the attention of different researchers, and it is a topic of current interest, see [1-28] and the references therein. Problems with integral boundary conditions come naturally from thermal conduction problems [29] and hydrodynamic problems [30]. In recent years there has been

(c) The Author(s) 2019. This article is distributed under the terms of the Creative Commons Attribution 4.0 International License (http://creativecommons.org/licenses/by/4.0/), which permits unrestricted use, distribution, and reproduction in any medium, provided you give appropriate credit to the original author(s) and the source, provide a link to the Creative Commons license, and indicate if changes were made. 
a lot of investigation of boundary value problems with integral boundary conditions (see for instance [31-43]). In particular, Boucherif [44] used the fixed point theorem in cones to consider the following problem:

$$
\left\{\begin{array}{l}
u^{\prime \prime}(t)=f(t, u(t)), \quad 0<t<1 \\
u(0)-c u^{\prime}(0)=\int_{0}^{1} g_{0}(t) u(t) d t \\
u(1)-d u^{\prime}(1)=\int_{0}^{1} g_{1}(t) u(t) d t
\end{array}\right.
$$

The author obtained several excellent results on the existence of positive solutions to problem (1.2).

Recently, Feng [45] studied the following boundary value problem:

$$
\left\{\begin{array}{l}
\left(g(t) x^{\prime}(t)\right)^{\prime}+w(t) f(t, x(t))=0, \quad 0<t<1, \\
a x(0)-b \lim _{t \rightarrow 0^{+}} g(t) x^{\prime}(t)=\int_{0}^{1} h(s) x(s) d s, \\
a x(1)+b \lim _{t \rightarrow 1^{-}} g(t) x^{\prime}(t)=\int_{0}^{1} h(s) x(s) d s .
\end{array}\right.
$$

The author got the existence results of symmetric positive solutions to problem (1.3) by applying the theory of fixed point index in cones. For other related results on problem (1.1), we refer the reader to [46-61] and the references cited therein.

At the same time, we notice that a type of problem on sharp conditions has received much attention, for instance, see [62-69] and the references cited therein. Specially, by the compressing fixed point theorem, Yang [65] gave the sharp conditions for the existence of positive solutions for the following second-order differential equation:

$$
\left\{\begin{array}{l}
u^{\prime \prime}(t)+f(u(t))=0, \quad 0<t<1 \\
\alpha u(0)-\beta u^{\prime}(0)=0 \\
\gamma u(1)+\delta u^{\prime}(1)=0
\end{array}\right.
$$

where $\alpha, \beta, \gamma, \delta \geq 0, \rho=\alpha \beta+\alpha \delta+\gamma \beta>0, f$ is singular at $t=0$ or $t=1$.

In [66], Pouso considered the following initial value problem:

$$
\left\{\begin{array}{l}
u^{\prime \prime}(t)=f(u(t)) \\
u(0)=u_{0}, \quad u^{\prime}(0)=u_{1}
\end{array}\right.
$$

The author obtained sharp conditions for local and global uniqueness and for the existence of periodic solutions for the above problem which is based on a detailed analysis of time maps. The other recent results concerning sharp condition problems can be found in [7075].

However, as we know, in literature there are no articles on sharp conditions for the analogous second order singular differential equations with integral boundary conditions. This shows that the study in the case of $\omega \in L^{p}[0,1]$ and $g \not \equiv 0$ is still open for problem (1.1). The purpose of this paper is to establish sharp conditions over $\omega$ and $f$ for the existence of positive solutions of (1.1). More precisely, we will investigate and give sharp conditions on the functions $\omega(t)$ and $f(t, u)$ which satisfy 
$\left(H_{4}\right) f(t, 1)>0, t \in J$, and there exist constants $\lambda_{1} \geq \lambda_{2}>1$ and $0<\lambda_{3} \leq \lambda_{4}<1$ such that, for all $t \in J, u \in[0,+\infty)$,

$$
\begin{array}{ll}
l^{\lambda_{1}} f(t, u) \leq f(t, l u) \leq l^{\lambda_{2}} f(t, u), & \forall l \in J^{\prime}=[0,1] ; \\
l^{\lambda_{4}} f(t, u) \leq f(t, l u) \leq l^{\lambda_{3}} f(t, u), & \forall l \in J^{\prime} .
\end{array}
$$

$\left(H_{5}\right)$

$$
0<\int_{0}^{1} H(s, s) f(s, 1) d s<+\infty
$$

where $H(s, s)$ is defined in (2.2).

Remark 1.1 It is not difficult to see that

(i) (1.4) is equivalent to

$$
l^{\lambda_{2}} f(t, u) \leq f(t, l u) \leq l^{\lambda_{1}} f(t, u), \quad \forall l \geq 1
$$

(ii) (1.5) is equivalent to

$$
l^{\lambda_{3}} f(t, u) \leq f(t, l u) \leq l^{\lambda_{4}} f(t, u), \quad \forall l \geq 1
$$

Remark 1.2 If $f(t, u)$ satisfies $\left(H_{4}\right)$, then it follows from (1.4) that, for every $t \in J, f(t, u)$ is nondecreasing with regard to $u \in[0,+\infty)$, and

$$
\lim _{u \rightarrow+\infty} \min _{t \in[\xi, \eta]} \frac{f(t, u)}{u}=+\infty, \quad \forall[\xi, \eta] \subset J .
$$

Similarly by (1.5), for every $t \in J, f(t, u)$ is nondecreasing with regard to $u \in[0,+\infty)$, and

$$
\lim _{u \rightarrow 0} \max _{t \in[\xi, \eta]} \frac{f(t, u)}{u}=0, \quad \forall[\xi, \eta] \subset J .
$$

The rest of the present paper is structured as follows. In the next section, we introduce some notation and preliminary results. In particular, we give some properties of the Green's function related to problem (1.1). In Sect. 3, by applying Hölder's inequality and combining the fixed point theorem, we analyze the sharp conditions for the existence of positive solutions for problem (1.1). Finally, in Sect. 4, we present a few of related remarks and comments.

\section{Preliminaries}

In this part, we prove a few lemmas and collect some known results for the convenience of later use and reference. The following definitions can be found in Guo and Lakshmikantham [76], or in Papageorgiou, Rădulescu, and Repovs [77].

Definition 2.1 Let $E$ be a real Banach space over $R$. A nonempty closed set $K \subset E$ is said to be a cone provided that

(i) $a^{\prime} u+b^{\prime} v \in K$ for all $u, v \in K$ and all $a^{\prime} \geq 0, b^{\prime} \geq 0$, and 
(ii) $u,-u \in K$ implies $u=0$.

Every cone $K \subset E$ induces an ordering in $E$ given by $u \leq v$ if and only if $v-u \in K$.

Lemma 2.1 Assume that $\left(H_{1}\right)-\left(H_{3}\right)$ hold and $\mu \neq 1$. Then, for any $y \in E$, the boundary value problem

$$
\left\{\begin{array}{l}
-u^{\prime \prime}(t)=y(t), \quad 0<t<1 \\
u(0)=u(1)=\int_{0}^{1} g(t) u(t) d t
\end{array}\right.
$$

has a unique solution u given by

$$
u(t)=\int_{0}^{1} H(t, s) y(s) d s
$$

where

$$
\begin{aligned}
& H(t, s)=G(t, s)+\frac{1}{1-\mu} \int_{0}^{1} G(\tau, s) g(\tau) d \tau, \\
& G(t, s)= \begin{cases}s(1-t), & 0 \leq s \leq t \leq 1 \\
t(1-s), & 0 \leq t \leq s \leq 1 .\end{cases}
\end{aligned}
$$

Proof First, suppose that $u$ is a solution of (2.1). It is easy to see by integration of (2.1) that

$$
u^{\prime}(t)-u^{\prime}(0)=-\int_{0}^{t} y(s) d s
$$

This shows

$$
u^{\prime}(t)=u^{\prime}(0)-\int_{0}^{t} y(s) d s .
$$

Integrating again, we obtain

$$
\begin{aligned}
u(t) & =u(0)+u^{\prime}(0) t-\int_{0}^{t} \int_{0}^{\tau} y(s) d s d \tau \\
& =u(0)+u^{\prime}(0) t-\int_{0}^{t} \int_{s}^{t} y(s) d \tau d s \\
& =u(0)+u^{\prime}(0) t-\int_{0}^{t} y(s)(t-s) d s
\end{aligned}
$$

Letting $t=1$ in (2.6), we get

$$
u(1)=u(0)+u^{\prime}(0)-\int_{0}^{1}(1-s) y(s) d s
$$

Combining the boundary condition $u(0)=u(1)$, we find

$$
u^{\prime}(0)=\int_{0}^{1}(1-s) y(s) d s
$$


Substituting the boundary condition $u(0)=\int_{0}^{1} g(t) u(t) d t$ and (2.8) into (2.6), we get

$$
\begin{aligned}
u(t) & =\int_{0}^{1} g(t) u(t) d t+\int_{0}^{1}(1-s) y(s) d s t-\int_{0}^{t}(t-s) y(s) d s \\
& =\int_{0}^{1} g(t) u(t) d t+\int_{0}^{t} s(1-t) y(s) d s+\int_{t}^{1} t(1-s) y(s) d s \\
& =\int_{0}^{1} g(t) u(t) d t+\int_{0}^{1} G(t, s) y(s) d s,
\end{aligned}
$$

where $G(t, s)$ is defined by (2.4). Multiplying the above equation by $g(t)$ and integrating it again, we obtain

$$
\begin{aligned}
\int_{0}^{1} g(t) u(t) d t & =\int_{0}^{1} g(t)\left[\int_{0}^{1} g(t) u(t) d t+\int_{0}^{1} G(t, s) y(s) d s\right] d t \\
& =\int_{0}^{1} g(t) u(t) d t \int_{0}^{1} g(t) d t+\int_{0}^{1} g(t) G(t, s) d t \int_{0}^{1} y(s) d s
\end{aligned}
$$

Then we have

$$
(1-\mu) \int_{0}^{1} g(t) u(t) d t=\int_{0}^{1} g(t) G(t, s) d t \int_{0}^{1} y(s) d s
$$

and

$$
\int_{0}^{1} g(t) u(t) d t=\frac{\int_{0}^{1} g(t) G(t, s) d t \int_{0}^{1} y(s) d s}{1-\mu} .
$$

Therefore, we have

$$
\begin{aligned}
u(t) & =\frac{1}{1-\mu} \int_{0}^{1} g(\tau) d \tau \int_{0}^{1} y(s) G(\tau, s) d s+\int_{0}^{1} G(t, s) y(s) d s \\
& =\int_{0}^{1}\left[G(t, s)+\frac{1}{1-\mu} \int_{0}^{1} g(\tau) G(\tau, s) d \tau\right] y(s) d s .
\end{aligned}
$$

Let

$$
H(t, s)=G(t, s)+\frac{1}{1-\mu} \int_{0}^{1} G(\tau, s) g(\tau) d \tau .
$$

Then

$$
u(t)=\int_{0}^{1} H(t, s) y(s) d s .
$$

The proof of Lemma 2.1 is complete.

We can show that $G(t, s)$ and $H(t, s)$ have the following properties.

Lemma 2.2 Let $\theta \in\left(0, \frac{1}{2}\right)$ and define $J_{0}=[\theta, 1-\theta]$. If $\mu \in[0,1)$, then for all $t \in J_{0}, s \in J^{\prime}$, we have

$$
0 \leq \theta e(s) \leq G(t, s) \leq e(s) \leq \frac{1}{4},
$$




$$
\begin{aligned}
& H(t, s) \leq H(s, s) \leq \gamma e(s) \leq \frac{1}{4} \gamma, \\
& H(t, s) \geq \theta H(s, s), \quad H(t, s) \geq \theta \gamma e(s),
\end{aligned}
$$

where

$$
\gamma=\frac{1}{1-\mu}, \quad e(s)=s(1-s) .
$$

Proof It is clear to see from the definition of $G(t, s)$ that $G(t, s) \leq e(s) \leq \frac{1}{4}$. Now, we show that $\theta e(s) \leq G(t, s)$ also holds.

In fact, for any $t \in J_{0}, s \in J^{\prime}$, we have the following.

Case 1. If $0<s \leq t \leq 1-\theta$, then

$$
\frac{G(t, s)}{G(s, s)}=\frac{s(1-t)}{s(1-s)}=\frac{1-t}{1-s} \geq 1-t \geq \theta .
$$

Case 2. If $\theta \leq t \leq s<1$, then

$$
\frac{G(t, s)}{G(s, s)}=\frac{t(1-s)}{s(1-s)}=\frac{t}{s} \geq t \geq \theta
$$

Case 3. If $s \in\{0,1\}$, then it naturally follows from the definition of $G(t, s)$ and $G(s, s)$ that $G(t, s)=G(s, s)=0$.

This shows that

$$
\theta e(s) \leq G(t, s), \quad t \in J_{0}, s \in J^{\prime} .
$$

It is not difficult to see that the inequality $H(t, s) \leq H(s, s)$ holds. Next, we show that $H(s, s) \leq \gamma e(s)$ also holds.

$$
\begin{aligned}
H(s, s) & \leq G(s, s)+\frac{1}{1-\mu} \int_{0}^{1} G(s, s) g(\tau) d \tau \\
& =G(s, s)\left[1+\frac{1}{1-\mu} \int_{0}^{1} g(\tau) d \tau\right] \\
& =\gamma e(s) .
\end{aligned}
$$

Therefore, the proof of (2.15) is complete.

Due to (2.14), we find

$$
\begin{aligned}
& H(t, s) \geq \theta G(s, s)+\frac{\theta}{1-\mu} \int_{0}^{1} G(s, s) g(\tau) d \tau \geq \theta H(s, s) \\
& H(t, s) \geq \theta G(s, s)\left[1+\frac{\int_{0}^{1} g(\tau) d \tau}{1-\mu}\right]=\frac{\theta}{1-\mu} G(s, s)=\theta \gamma e(s) .
\end{aligned}
$$

So, for all $t \in J_{0}, s \in J^{\prime}$, (2.16) is established.

This concludes the proof of Lemma 2.2. 
Definition 2.2 If a function $u$ satisfies (1.1) and $u(t)>0, t \in J$, then it is said that $u \in$ $C[0,1] \cap C^{2}(0,1)$ is a positive solution of problem $(1.1)$; If the positive solution $u \in C^{1}[0,1]$, namely $u^{\prime}\left(0^{+}\right)$and $u^{\prime}\left(1^{-}\right)$exist, then $u$ is said to be a $C^{1}[0,1]$ positive solution of problem (1.1).

Let $E=C[0,1]$. Then $E$ is a real Banach space with norm $\|\cdot\|$ defined by

$$
\|u\|=\sup _{t \in J^{\prime}}|u(t)|, \quad u \in E
$$

To establish the existence of positive solutions to problem (1.1), we consider the cone $K$ defined by

$$
K=\left\{u \in E: u(t) \geq \theta\|u\|, t \in J^{\prime}\right\}
$$

where $\theta$ is a constant as in Lemma 2.2. It is easy to see that $K$ is a convex cone of $E$.

Also, define, for a given positive number $r$, the set $\Omega_{r}$ by

$$
\begin{aligned}
& \Omega_{r}=\{u \in K:\|u\|<r\}, \\
& \partial \Omega_{r}=\{u \in K:\|u\|=r\} .
\end{aligned}
$$

To get some norm inequalities in our main results, we employ Hölder's inequality.

Lemma 2.3 (Hölder) Let $e \in L^{p}[a, b]$ with $p>1, h \in L^{q}[a, b]$ with $q>1$, and $\frac{1}{p}+\frac{1}{q}=1$. Then $e h \in L^{1}[a, b]$, and

$$
\|e h\|_{1} \leq\|e\|_{p}\|h\|_{q}
$$

Let $e \in L^{1}[a, b]$ and $h \in L^{\infty}[a, b]$. Then $e h \in L^{1}[a, b]$ and

$$
\|e h\|_{1} \leq\|e\|_{1}\|h\|_{\infty}
$$

Lemma 2.4 Assume that $\left(H_{1}\right)-\left(H_{5}\right)$ hold. Define $T: K \rightarrow E$ by

$$
(T u)(t)=\int_{0}^{1} H(t, s) \omega(s) f(s, u(s)) d s, \quad \forall u \in K
$$

Then $u \in C[0,1]$ is a $C[0,1] \cap C^{2}(0,1)$ positive solution of $(1.1)$ if and only if $u$ is a fixed point of $T$.

Proof Suppose that $\left(H_{1}\right)-\left(H_{5}\right)$ hold. For fixed $u \in E, u(t) \geq 0, t \in J^{\prime}$, taking a constant $0<a<1$ such that $a\|u\|<1$, then it follows from (1.4) and (1.5) that

$$
f(t, u(t)) \leq\left(\frac{1}{a}\right)^{\lambda_{1}} f(t, a u(t)) \leq\left(\frac{1}{a}\right)^{\lambda_{1}} f(t, a\|u\|) \leq a^{\lambda_{2}-\lambda_{1}}\|u\|^{\lambda_{2}} f(t, 1)
$$

Consequently, for all $t \in J^{\prime}$, we get

$$
0<\int_{0}^{1} H(t, s) \omega(s) f(s, u(s)) d s \leq a^{\lambda_{2}-\lambda_{1}}\|u\|^{\lambda_{2}}\|\omega\|_{1} \int_{0}^{1} H(s, s) f(s, 1) d s<+\infty .
$$


It is obvious that the operator

$$
(T u)(t)=\int_{0}^{1} H(t, s) \omega(s) f(s, u(s)) d s, \quad \forall u \in K
$$

is defined well. And hence the definition of $T$ and the properties of $G(t, s)$ and $H(t, s)$ yield that $u \in C[0,1]$ is a $C[0,1] \cap C^{2}(0,1)$ positive solution of $(1.1)$ if and only if $u$ is a positive fixed point of operator $T$. This finishes the proof of Lemma 2.4.

Lemma 2.5 ((Theorem 2.3.4 of [76]) (Fixed point theorem of cone expansion and compression of norm type)) Let $\Omega_{1}$ and $\Omega_{2}$ be two bounded open sets in a real Banach space E such that $0 \in \Omega_{1}$ and $\bar{\Omega}_{1} \subset \Omega_{2}$. Let the operator $T: K \cap\left(\bar{\Omega}_{2} \backslash \Omega_{1}\right) \rightarrow K$ be completely continuous, where $K$ is a cone in E. Suppose that one of the two conditions

(i) $\|T x\| \leq\|x\|, \forall x \in K \cap \partial \Omega_{1}$ and $\|T x\| \geq\|x\|, \forall x \in K \cap \partial \Omega_{2}$, and

(ii) $\|T x\| \geq\|x\|, \forall x \in K \cap \partial \Omega_{1}$, and $\|T x\| \leq\|x\|, \forall x \in K \cap \partial \Omega_{2}$, is satisfied. Then $T$ has at least one fixed point in $K \cap\left(\bar{\Omega}_{2} \backslash \Omega_{1}\right)$.

Lemma 2.6 If $u$ is a $C^{1}[0,1]$ positive solution of problem (1.1), then there exists $b>0$ satisfying $u(s) \geq b H(s, s), s \in J^{\prime}$.

Proof Let $u$ take its maximum at $t_{0}$. Then we discuss Lemma 2.6 under the following two cases.

Case 1 . If $0<t_{0} \leq \frac{1}{2}$, then let

$$
h(t)= \begin{cases}\frac{1-t_{0}}{t_{0}} t, & 0 \leq t \leq t_{0} \\ -t+1, & t_{0}<t \leq 1 .\end{cases}
$$

Case 2. If $\frac{1}{2}<t_{0}<1$, then let

$$
h(t)=\left\{\begin{array}{l}
t, \quad 0 \leq t \leq t_{0}, \\
-\frac{t_{0}}{1-t_{0}} t+\frac{t_{0}}{1-t_{0}}, \quad t_{0}<t \leq 1 .
\end{array}\right.
$$

Due to the concavity of $u$ and since $h\left(t_{0}\right)<1$, we have

$$
u(t) \geq u\left(t_{0}\right) h(t)
$$

Next we show that $h(t)>e(t)$ on $J^{\prime}$ holds.

It is easy to see by calculating that

$$
e^{\prime}(0)=1, \quad e^{\prime}(1)=-1 .
$$

On the one hand, when $0<t_{0} \leq \frac{1}{2}$, we have

$$
h^{\prime}(t)= \begin{cases}\frac{1-t_{0}}{t_{0}}, & 0 \leq t \leq t_{0} \\ -1, & t_{0}<t \leq 1\end{cases}
$$


It is obvious that

$$
\frac{1-t_{0}}{t_{0}}>1
$$

so by the concavity of $e$ we have that $h(t)>e(t)$ on $J^{\prime}$.

On the other hand, when $\frac{1}{2}<t_{0}<1$, we have

$$
h^{\prime}(t)=\left\{\begin{array}{l}
1, \quad 0 \leq t \leq t_{0} \\
-\frac{1-t_{0}}{t_{0}}, \quad t_{0}<t \leq 1 .
\end{array}\right.
$$

It can be easily seen that

$$
-\frac{1-t_{0}}{t_{0}}<-1
$$

similarly we can obtain that $h(t)>e(t)$ on $J^{\prime}$.

At the same time, by Lemma 2.2, we have

$$
u(t) \geq u\left(t_{0}\right) h(t) \geq u\left(t_{0}\right) e(t) \geq \frac{u\left(t_{0}\right)}{\gamma} H(t, t)=b H(t, t), \quad t \in J
$$

where $b=\frac{u\left(t_{0}\right)}{\gamma}$.

In order to better understand the above two cases, we draw Figs. 1 and 2.

This gives the proof of Lemma 2.6.

Figure 1 Case 1

Figure 2 Case 2
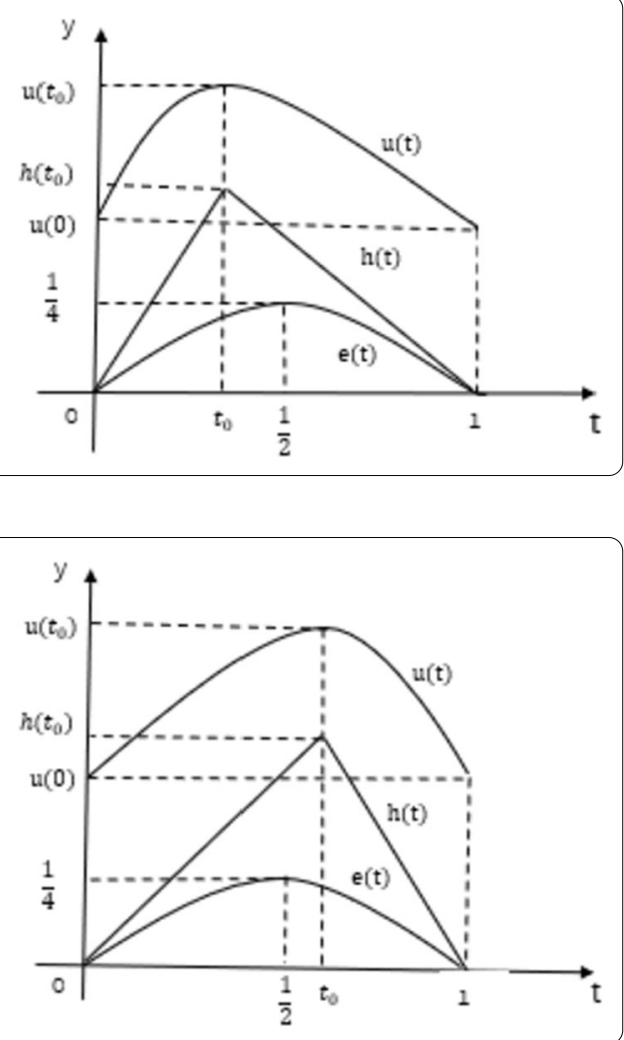


\section{Sharp conditions for the existence of positive solutions}

In this section, we establish sharp conditions for the existence of positive solutions for problem (1.1) by Lemmas 2.1-2.6. We analyze the following three cases for $\omega \in L^{p}[0,1]$ : $p>1, p=1$, and $p=\infty$. Case $p>1$ is treated in Theorem 3.1.

Theorem 3.1 Suppose that $\left(H_{1}\right)-\left(H_{5}\right)$ hold. Then problem $(1.1)$ admits a $u \in C^{1}[0,1]$ positive solution if and only if

$$
0<\int_{0}^{1} \omega(s) f(s, 1) d s<+\infty
$$

Proof (1) Necessity.

Let $u \in C^{1}[0,1]$ be a positive solution of problem (1.1), then $u^{\prime}(0)$ and $u^{\prime}(1)$ exist and are finite.

On the one hand, we know that $u(t)$ is a concave function on $J^{\prime}$ by $u^{\prime \prime} \leq 0$. Therefore, by Lemma 2.6, there exists $b>0$ satisfying $u(s) \geq b H(s, s), s \in J^{\prime}$. Setting $l=\min \{b, 1\}$, then $u(s) \geq l H(s, s), s \in J^{\prime}$. And by (1.4) and Remark 1.2, we have

$$
\begin{aligned}
\int_{0}^{1} \omega(s) f(s, H(s, s)) d s & \leq \int_{0}^{1} \omega(s) f\left(s, \frac{1}{l} u(s)\right) d s \\
& \leq \bar{l} \int_{0}^{1}\left[-u^{\prime \prime}(s)\right] d s \\
& \leq \bar{l}\left[u^{\prime}(0)-u^{\prime}(1)\right] \\
& <+\infty
\end{aligned}
$$

where $\bar{l}=\left(\frac{1}{l}\right)^{\lambda_{1}}$.

On the other hand, if we assume that $f(s, u(s)) \equiv 0$, which shows that $\omega(s) f(s, u(s)) \equiv 0$ by $\left(H_{1}\right)$. Then, by Lemma 2.4 , it is obvious that $u=0$, which contradicts $u$ is a positive solution of problem (1.1).

Hence there exists $t_{1} \in J$ such that $f\left(t_{1}, u\left(t_{1}\right)\right)>0$.

And then it follows from $\left(H_{1}\right)$, Remarks 1.2 and (1.6) that

Case 1. If $\omega\left(t_{1}\right)>0$, then

$$
\begin{aligned}
0 & <\omega\left(t_{1}\right) f\left(t_{1}, u\left(t_{1}\right)\right) \\
& \leq \omega\left(t_{1}\right) f\left(t_{1},\left\|u_{t_{1}}\right\|\right) \\
& \leq \omega\left(t_{1}\right)\left\|u_{t_{1}}\right\|^{\lambda} f\left(t_{1}, 1\right),
\end{aligned}
$$

where $\lambda \in\left\{\lambda_{2}, \lambda_{3}\right\}$.

Case 2. If $\omega\left(t_{1}\right)=0$, then it follows from $\left(H_{1}\right)$ that there exists a small neighborhood $\left[a_{1}, b_{1}\right] \subset J$ of $t_{1}$ such that $\omega(t)>0$ for $t \in\left[a_{1}, b_{1}\right]$.

Hence it is easy to see by integration of $f$ and $\omega$ that

$$
\int_{a_{1}}^{b_{1}} \omega(s) f(s, 1) d s>0
$$


So,

$$
\begin{aligned}
\int_{0}^{1} \omega(s) f(s, 1) d s & \geq \int_{0}^{1} \omega(s) f\left(s, \frac{u(s)}{\|u\|}\right) d s \\
& \geq\left(\frac{1}{\|u\|}\right)^{\lambda^{*}} \int_{0}^{1} \omega(s) f(s, u(s)) d s \\
& \geq\left(\frac{1}{\|u\|}\right)^{\lambda^{*}} \int_{a_{1}}^{b_{1}} \omega(s) f(s, u(s)) d s \\
& >0,
\end{aligned}
$$

where $\lambda^{*} \in\left\{\lambda_{1}, \lambda_{4}\right\}$.

(2) Sufficiency.

(i) First, we prove that the operator $T: K \rightarrow K$ is completely continuous. For all $u \in K$, $T(u) \geq 0$ on $J_{0}$, it follows from (2.18) and Lemma 2.2 that

$$
\begin{aligned}
(T u)(t) & =\int_{0}^{1} H(t, s) \omega(s) f(s, u(s)) d s \\
& \geq \theta \int_{0}^{1} H(t, s) \omega(s) f(s, u(s)) d s \\
& =\theta\|T y\|, \quad \forall t \in J^{\prime} .
\end{aligned}
$$

So we have that $T u \in K, \forall u \in K$. Thus $T(K) \subset K$.

Next, it follows from Arzelà-Ascoli theorem that $T: K \rightarrow K$ is completely continuous. It is clear that $T$ is continuous.

Let $B_{r}=\{u \in E \mid\|u\| \leq r\}$ be a bounded set. Then, for all $u \in B_{r}$, by the definition of $\|T u\|$ and by Lemma 2.2 and Lemma 2.3, we get

$$
\begin{aligned}
\|T u\| & =\max _{t \in J} \int_{0}^{1} H(t, s) \omega(s) f(s, u(s)) d s \\
& \leq \gamma \int_{0}^{1} G(s, s) \omega(s) d s L \\
& \leq \gamma\|G\|_{q}\|\omega\|_{p} L \\
& =\Gamma
\end{aligned}
$$

where $L=\max _{t \in J, u \in B_{r}} f(t, u), \Gamma=\gamma\|G\|_{q}\|\omega\|_{p} L$.

Therefore, the operator $T: K \longrightarrow K$ is uniformly bounded.

On the other hand, since $H(t, s)$ is continuous on $J^{\prime} \times J^{\prime}$, we can see that $H(t, s)$ is uniformly continuous on $J^{\prime} \times J^{\prime}$. Therefore, for any $\varepsilon>0$, there exists $r>0$, when $\left|t_{1}-t_{2}\right|<r$, we get

$$
\left|H\left(t_{1}, s\right)-H\left(t_{2}, s\right)\right|<\frac{\varepsilon}{\|\omega\|_{1} \cdot L}
$$


Accordingly, for all $u \in B_{r}$, when $\left|t_{1}-t_{2}\right|<r$, we have

$$
\begin{aligned}
\left|(T u)\left(t_{1}\right)-(T u)\left(t_{2}\right)\right| & =\left|\int_{0}^{1} H\left(t_{1}, s\right) \omega(s) f(s, u(s)) d s-\int_{0}^{1} H\left(t_{2}, s\right) \omega(s) f(s, u(s)) d s\right| \\
& =\left|\int_{0}^{1}\left[H\left(t_{1}, s\right)-H\left(t_{2}, s\right)\right] \omega(s) f(s, u(s)) d s\right| \\
& \leq\left|\|\omega\|_{1} \cdot L\right| \cdot \frac{\varepsilon}{\|\omega\|_{1} \cdot L} \\
& \leq \varepsilon .
\end{aligned}
$$

This shows that the set $\left\{T(u): u \in B_{r}\right\}$ is equicontinuous, and it follows from ArzelàAscoli theorem that operator $T$ is completely continuous.

(ii) Next, we prove that $T$ has at least one fixed point in $K$.

For $u \in K,\|u\| \leq 1$, we get $u(t) \leq\|u\| \leq 1$, and by (1.4) and Remark 1.2, we obtain

$$
f(t, u(t)) \leq u^{\lambda_{2}}(t) f(t, 1)
$$

Hence,

$$
\begin{aligned}
\|T u\| & \leq \int_{0}^{1} \gamma G(s, s) \omega(s) u^{\lambda_{2}}(s) f(s, 1) d s \\
& \leq \gamma\|u\|^{\lambda_{2}}\|G\|_{q}\|\omega\|_{p} \int_{0}^{1} f(s, 1) d s \\
& \leq A\|u\|^{\lambda_{2}},
\end{aligned}
$$

where $A=\gamma\|G\|_{q}\|\omega\|_{p} \int_{0}^{1} f(s, 1) d s$.

If $\left(\frac{1}{A}\right)^{\frac{1}{\lambda^{-1}}} \leq 1$, setting $r_{1}^{*}=\left(\frac{1}{A}\right)^{\frac{1}{\lambda_{2}-1}}$, then $\|T u\| \leq\|u\|, \forall u \in K,\|u\|=r_{1}^{*}$.

If $\left(\frac{1}{A}\right)^{\frac{1}{\lambda_{2}-1}}>1$, we have $A<1$. Letting $r_{1}^{* *}=1$, similarly we have $\|T u\| \leq\|u\|, \forall u \in K$, $\|u\|=r_{1}^{* *}$.

Set $r_{1}=\max \left\{r_{1}^{*}, r_{1}^{* *}\right\}$. Then we obtain $\|T u\| \leq\|u\|, \forall u \in K,\|u\|=r_{1}$.

Moreover, by Remark 1.2, there exists $R>r_{1}$ for $u \geq R$ such that

$$
\frac{f(t, u(t))}{u(t)} \geq \min _{t \in J_{0}} \frac{f(t, u(t))}{u(t)} \geq N, \quad t \in J_{0},
$$

that is, $f(t, u(t)) \geq N u(t), t \in J_{0}, u \geq R$, where $N>0$ satisfies

$$
N \geq \frac{1}{\zeta \theta \min _{t \in J_{0}} \int_{\theta}^{1-\theta} H(t, s) d s}
$$

So, for $u \in K$ with $\|u\|=R$, we get

$$
\begin{aligned}
\|T u\| & \geq \min _{t \in J_{0}}(T u)(t) \geq \min _{t \in J_{0}} \int_{\theta}^{1-\theta} H(t, s) \omega(s) f(s, u(s)) d s \\
& \geq \min _{t \in J_{0}} \int_{\theta}^{1-\theta} H(t, s) \zeta N u(s) d s
\end{aligned}
$$




$$
\begin{aligned}
& \geq N \zeta \min _{t \in J_{0}} \int_{\theta}^{1-\theta} H(t, s)\|u\| \theta d s \\
& \geq N \zeta \theta \min _{t \in J_{0}} \int_{\theta}^{1-\theta} H(t, s) d s\|u\| \\
& \geq\|u\| .
\end{aligned}
$$

Thus, $\|T u\| \geq\|u\|, \forall u \in K,\|u\|=R$.

Lemma 2.5 yields that $T$ admits at least one fixed point $u^{*}$ such that $r_{1} \leq\left\|u^{*}\right\| \leq R$. Since $u^{*}(t) \geq\left\|u^{*}\right\| \theta \geq r_{1} \theta>0,0<t<1$, we see that $u^{*}$ is a positive solution of problem (1.1).

Moreover, for any $u^{*} \in K$, we have $u^{*}(s) \leq\left\|u^{*}\right\|, s \in J^{\prime}$, and then, for $\lambda \in\left\{\lambda_{2}, \lambda_{3}\right\}$, we get

$$
\begin{aligned}
\int_{0}^{1}\left|\left(u^{*}\right)^{\prime \prime}(s)\right| d s & =\int_{0}^{1} \omega(s) f\left(s, u^{*}(s)\right) d s \\
& \leq \int_{0}^{1} \omega(s) f\left(s,\left\|u^{*}\right\|\right) d s \\
& \leq\left\|u^{*}\right\|^{\lambda} \int_{0}^{1} \omega(s) f(s, 1) d s \\
& <+\infty,
\end{aligned}
$$

that is, $u^{*}$ is absolutely integrable on $J^{\prime}$. This shows that $\left(u^{*}\right)^{\prime}\left(0^{+}\right)$and $\left(u^{*}\right)^{\prime}\left(1^{-}\right)$exist, then $u^{*} \in C^{1}[0,1]$. The proof above shows that $u^{*} \in C^{1}[0,1]$ is a positive solution of (1.1). This completes the proof of Theorem 3.1.

The following corollary handles the case $p=\infty$.

Corollary 3.1 Assume that $\left(H_{1}\right)-\left(H_{5}\right)$ hold. Then problem $(1.1)$ admits a $u \in C^{1}[0,1]$ positive solution if and only if

$$
0<\int_{0}^{1} \omega(s) f(s, 1) d s<+\infty
$$

Proof Let $\|G\|_{1}\|\omega\|_{\infty}$ replace $\|G\|_{q}\|\omega\|_{p}$ and repeat the argument of Theorem 3.1. Then we can complete the proof of Corollary 3.1.

At last, we analyze the case of $p=1$.

Corollary 3.2 Assume that $\left(H_{1}\right)-\left(H_{5}\right)$ hold. Then problem $(1.1)$ has a $u \in C^{1}[0,1]$ positive solution if and only if

$$
0<\int_{0}^{1} \omega(s) f(s, 1) d s<+\infty
$$

Proof Let $\frac{1}{4}\|\omega\|_{1}$ replace $\|G\|_{q}\|\omega\|_{p}$ and repeat the argument of Theorem 3.1. Then we can complete the proof of Corollary 3.2.

The following theorem only considers the case of $p>1$. 
Theorem 3.2 Assume $f(t, u)=h_{1}(t, u)+h_{2}(t, u)$, where $h_{1}(t, u)$ and $h_{2}(t, u)$ satisfy $\left(H_{4}\right)$, and the other main hypothesis is also needed

$$
0<\gamma\|G\|_{q}\|\omega\|_{p} \int_{0}^{1} f(s, 1) d s<1
$$

Then problem (1.1) admits at least two $C^{1}[0,1]$ positive solutions if and only if

$$
0<\int_{0}^{1} \omega(s) f(s, 1) d s<+\infty .
$$

Proof We first prove that the operator

$$
\begin{aligned}
(T u)(t) & =\int_{0}^{1} H(t, s) \omega(s) f(s, u(s)) d s \\
& =\int_{0}^{1} H(t, s) \omega(s)\left[h_{1}(s, u(s))+h_{2}(s, u(s))\right] d s
\end{aligned}
$$

admits at least two fixed points in $K$.

Choosing $J_{1}=\left[\xi_{1}, \eta_{1}\right] \subset J, \tau_{1}=\min _{t \in J_{1}} h_{1}(t, 1)$ and taking a constant $l>1$ such that $l \theta>1$, for $\|u\|>1, u \in K, t \in J_{1}$, we obtain

$$
l u(t) \geq l \theta\|u\|>l \theta>1, \quad h_{1}(t, u(t)) \geq l^{\lambda_{2}-\lambda_{1}} u(t)^{\lambda_{2}} h_{1}(t, 1) .
$$

Consequently,

$$
\begin{aligned}
(T u)(t) & \geq \int_{\xi_{1}}^{\eta_{1}} H(t, s) \omega(s)\left[h_{1}(s, u(s))+h_{2}(s, u(s))\right] d s \\
& \geq \int_{\xi_{1}}^{\eta_{1}} G(t, s) \omega(s)\left[h_{1}(s, u(s))+h_{2}(s, u(s))\right] d s \\
& \geq \theta^{\lambda_{2}} \tau_{1} l^{\left(\lambda_{2}-\lambda_{1}\right)} \zeta \int_{\xi_{1}}^{\eta_{1}} G\left(\xi_{1}, s\right) d s\|u\|^{\lambda_{2}} \\
& \geq A\|u\|^{\lambda_{2}},
\end{aligned}
$$

where $A=\theta^{\lambda_{2}} \tau_{1} l^{\left(\lambda_{2}-\lambda_{1}\right)} \zeta \int_{\xi_{1}}^{\eta_{1}} G\left(\xi_{1}, s\right) d s$.

Due to $\|u\|>1, \lambda_{2}>1$, there exists arbitrarily large $R_{2}>1$ such that

$$
\|T u\| \geq\|u\|, \quad \forall u \in K,\|u\|=R_{2} .
$$

When $\|u\|<1$, taking $J_{2}=\left[\xi_{2}, \eta_{2}\right] \subset J$ and $\tau_{2}=\min _{t \in J_{2}} h_{2}(t, 1)$, we also get that

$$
\begin{aligned}
(T u)(t) & \geq \int_{\xi_{2}}^{\eta_{2}} G(t, s) \omega(s)\left[h_{1}(s, u(s))+h_{2}(s, u(s))\right] d s \\
& \geq \theta^{\lambda_{4}} \tau_{2} \zeta \int_{\xi_{2}}^{\eta_{2}} G\left(\xi_{2}, s\right) d s\|u\|^{\lambda_{4}} \\
& \geq A_{1}\|u\|^{\lambda_{4}},
\end{aligned}
$$

where $A_{1}=\theta^{\lambda_{4}} \tau_{2} \zeta \int_{\xi_{2}}^{\eta_{2}} G\left(\xi_{2}, s\right) d s$. 
Similarly, due to $\|u\|<1, \lambda_{4}<1$, there exists arbitrarily small $r_{2}<1$ such that

$$
\|T u\| \geq\|u\|, \quad \forall u \in K,\|u\|=r_{2} .
$$

Moreover, because of $u \in K,\|u\|=1$ and $u(t) \leq\|u\|=1 \leq 1$, we can obtain

$$
h_{1}(t, u(t))+h_{2}(t, u(t)) \leq u(t)^{\lambda_{2}} h_{1}(t, 1)+u(t)^{\lambda_{3}} h_{2}(t, 1) \leq\left[h_{1}(s, 1)+h_{2}(s, 1)\right] .
$$

Accordingly,

$$
\begin{aligned}
(T u)(t) & \leq \int_{0}^{1} \gamma G(s, s) \omega(s)\left[h_{1}(s, u(s))+h_{2}(s, u(s))\right] d s \\
& \leq \gamma\|G\|_{q}\|\omega\|_{p} \int_{0}^{1}\left[h_{1}(s, 1)+h_{2}(s, 1)\right] d s \\
& <1=\|u\| .
\end{aligned}
$$

That is, $\|T u\|<\|u\|, \forall u \in K \cap \partial \Omega=\{u \in K:\|u\|=1\}$.

Consequently, Lemma 2.5 yields that the operator $T$ admits at least two fixed points $u_{1}(t)$ and $u_{2}(t)$ in $K$, and $u_{1}(t) \neq u_{2}(t)$ by (3.1).

On the other hand, the proof of necessity is similar to that of Theorem 3.1, so we omit it here. The proof of Theorem 3.2 is complete.

\section{Remarks and comments}

In this section, we provide some remarks and comments related to problem (1.1).

Remark 4.1 The proof of Theorems 3.1-3.2 is directly inspired by Theorem 1.1 of [63], but there are no papers analyzing sharp conditions of positive solution for second-order boundary value problems with integral boundary conditions, particularly under the case $\omega$ is $L^{p}$-integrable.

Remark 4.2 In general, it is difficult to analyze sharp conditions of positive solutions for nonlinear second-order differential equations (see, e.g., [1-28] and their references).

Remark 4.3 Similar to the proof of Theorems 3.1-3.2, one can prove sharp conditions of positive solution for the following problems:

$$
\begin{aligned}
& \left\{\begin{array}{l}
u^{\prime \prime}(t)+\omega(t) f(t, u(t))=0, \\
u(0)=\int_{0}^{1} g(t) u(t) d t, \quad u(1)=0,
\end{array}\right. \\
& \left\{\begin{array}{l}
u^{\prime \prime}(t)+\omega(t) f(t, u(t))=0, \\
u(0)=0, \quad u(1)=\int_{0}^{1} g(t) u(t) d t,
\end{array}\right.
\end{aligned}
$$

where $J=(0,1), \omega \in L^{p}[0,1]$ for some $1 \leq p \leq+\infty, f \in C\left(J \times R^{+}, R^{+}\right), R^{+}=[0,+\infty)$ (here, $f$ may be singular at $t=0$ and/or 1$), g \in L^{1}[0,1]$. 


\section{Acknowledgements}

We wish to express our thanks to Prof. Meiqiang Feng, School of Applied Science, Beijing Information Science \& Technology University, Beijing, PR China, for his kind help, careful reading, and making useful comments on the earlier version of this paper. The authors are also grateful to anonymous referees for their constructive comments and suggestions which have greatly improved this paper.

\section{Funding}

This work is sponsored by the National Natural Science Foundation of China (11301178) and the Beijing Natural Science Foundation of China (1163007)

\section{Availability of data and materials}

Not applicable.

\section{Ethics approval and consent to participate}

Not applicable.

\section{Competing interests}

The authors declare that there is no conflict of interests regarding the publication of this manuscript. The authors declare that they have no competing interests.

\section{Consent for publication}

Not applicable.

\section{Authors' contributions}

The authors contributed equally in this article. They have all read and approved the final manuscript.

\section{Publisher's Note}

Springer Nature remains neutral with regard to jurisdictional claims in published maps and institutional affiliations.

Received: 27 March 2019 Accepted: 21 October 2019 Published online: 30 October 2019

\section{References}

1. Hao, X., Zuo, M., Liu, L.: Multiple positive solutions for a system of impulsive integral boundary value problems with sign-changing nonlinearities. Appl. Math. Lett. 82, 24-31 (2018)

2. Mao, J., Zhao, Z.: The existence and uniqueness of positive solutions for integral boundary value problems. Bull. Malays. Math. Sci. Soc. 34, 153-164 (2011)

3. Jiao, L., Zhang, X.: A class of second-order nonlocal indefinite impulsive differential systems. Bound. Value Probl. 2018 $163(2018)$

4. Zhang, X.: Exact interval of parameter and two infinite families of positive solutions for a $n$th order impulsive singular equation. J. Comput. Appl. Math. 330, 896-908 (2018)

5. Hao, X., Liu, L., Wu, Y.: Positive solutions for second order impulsive differential equations with integral boundary conditions. Commun. Nonlinear Sci. Numer. Simul. 16, 101-111 (2011)

6. Wang, Y., Liu, L., Zhang, X., Wu, Y.: Positive solutions of an abstract fractional semipositone differential system model for bioprocesses of HIV infection. Appl. Math. Comput. 258, 312-324 (2015)

7. Autuori, G., Cluni, F., Gusella, V., Pucci, P.: Mathematical models for nonlocal elastic composite materials. Adv. Nonlinear Anal. 6, 355-382 (2017)

8. Zhang, X., Ge, W.: Symmetric positive solutions of boundary value problems with integral boundary conditions. Appl. Math. Comput. 219, 3553-3564 (2012)

9. Hao, X., Liu, L., Wu, Y.: Positive solutions for singular second order differential equations with integral boundary conditions. Math. Comput. Model. 57, 836-847 (2013)

10. Hao, X., Liu, L., Wu, Y., Sun, Q.: Positive solutions for nonlinear $n$ th-order singular eigenvalue problem with nonlocal conditions. Nonlinear Anal., Theory Methods Appl. 73, 1653-1662 (2010)

11. Hao, X., Wang, H., Liu, L., Cui, Y.: Positive solutions for a system of nonlinear fractional nonlocal boundary value problems with parameters and $p$-Laplacian operator. Bound. Value Probl. 2017, 182 (2017)

12. Zhang, X., Feng, M.: Existence of a positive solution for one-dimensional singular $p$-Laplacian problems and its parameter dependence. J. Math. Anal. Appl. 413, 566-582 (2014)

13. Hao, X., Xu, N., Liu, L.: Existence and uniqueness of positive solutions for fourth-order $m$-point boundary value problems with two parameters. Rocky Mt. J. Math. 43, 1161-1180 (2013)

14. Hao, X., Liu, L., Wu, Y.: On positive solutions of an $m$-point nonhomogeneous singular boundary value problem. Nonlinear Anal., Theory Methods Appl. 73, 2532-2540 (2010)

15. Zhang, X., Feng, M.: Double bifurcation diagrams and four positive solutions of nonlinear boundary value problems via time maps. Commun. Pure Appl. Anal. 17, 2149-2171 (2018)

16. Zhang, X., Liu, L., Wu, Y.: The uniqueness of positive solution for a fractional order model of turbulent flow in a porous medium. Appl. Math. Lett. 37, 26-33 (2014)

17. Zhong, Q., Zhang, X: Positive solution for higher-order singular infinite-point fractional differential equation with p-Laplacian. Adv. Differ. Equ. 2016, 11 (2016)

18. Dong, X., Bai, Z., Zhang, S.: Positive solutions to boundary value problems of $p$-Laplacian with fractional derivative. Bound. Value Probl. 2017, 5 (2017)

19. Hao, X. Liu, L.: Multiple monotone positive solutions for higher order differential equations with integral boundary conditions. Bound. Value Probl. 2014, 74 (2014) 
20. $\mathrm{Wu}$, J., Zhang, X., Liu, L., Wu, Y., Cui, Y.: Convergence analysis of iterative scheme and error estimation of positive solution for a fractional differential equation. Math. Model. Anal. 23, 611-626 (2018)

21. He, J., Zhang, X., Liu, L., Wu, Y., Cui, Y.: Existence and asymptotic analysis of positive solutions for a singular fractional differential equation with nonlocal boundary conditions. Bound. Value Probl. 2018, 189 (2018)

22. Zhang, X., Mao, C., Liu, L., Wu, Y.: Exact iterative solution for an abstract fractional dynamic system model for bioprocess. Qual. Theory Dyn. Syst. 16, 205-222 (2017)

23. Zhang, X., Zhong, Q.: Triple positive solutions for nonlocal fractional differential equations with singularities both on time and space variables. Appl. Math. Lett. 80, 12-19 (2018)

24. Hao, X., Zhang, L., Liu, L.: Positive solutions of higher order fractional integral boundary value problem with a parameter. Nonlinear Anal., Model. Control 24, 210-223 (2019)

25. Hao, X., Wang, H.: Positive solutions of semipositone singular fractional differential systems with a parameter and integral boundary conditions. Open Math. 16, 581-596 (2018)

26. Mao, J., Zhao, Z., Wang, C.: The exact iterative solution of fractional differential equation with nonlocal boundary value conditions. J. Funct. Spaces 2018, Article ID 8346398 (2018)

27. Guan, Y., Zhao, Z., Lin, X.: On the existence of positive solutions and negative solutions of singular fractional differential equations via global bifurcation techniques. Bound. Value Probl. 2016, 141 (2016)

28. Feng, M., Li, P., Sun, S.: Symmetric positive solutions for fourth-order $n$-dimensional $m$-Laplace systems. Bound. Value Probl. 2018, $63(2018)$

29. Cannon, J.R.: The solution of the heat equation subject to the specification of energy. Q. Appl. Math. 21, 155-160 (1963)

30. Chegis, R.Yu.: Numerical solution of a heat conduction problem with an integral boundary condition. Liet. Mat. Rink. 24, 209-215 (1984)

31. Qin, P., Feng, M., Li, P.: Positive solutions to one-dimensional quasilinear impulsive indefinite boundary value problems. Adv. Differ. Equ. 2018, 421 (2018)

32. Jiang, J., Liu, L., Wu, Y. Positive solutions for second order impulsive differential equations with Stieltjes integral boundary conditions. Adv. Differ. Equ. 2012, 124 (2012)

33. Zhang, X., Feng, M., Ge, W.: Existence result of second-order differential equations with integral boundary conditions at resonance. J. Math. Anal. Appl. 353, 311-319 (2009)

34. Zhang, X., Ge, W.: Symmetric positive solutions of boundary value problems with integral boundary conditions. Appl. Math. Comput. 219, 3553-3564 (2012)

35. Guo, L., Liu, L., Wu, Y.: Iterative unique positive solutions for singular $p$-Laplacian fractional differential equation system with several parameters. Nonlinear Anal., Model. Control 22, 182-203 (2018)

36. Zhang, X., Liu, L., Wu, Y., Wiwatanapataphee, B.: The spectral analysis for a singular fractional differential equation with a signed measure. Appl. Math. Comput. 257, 252-263 (2015)

37. Zhang, X., Liu, L., Wu, Y.: Variational structure and multiple solutions for a fractional advection-dispersion equation Comput. Math. Appl. 68, 1794-1805 (2014)

38. Zhang, X., Liu, L., Wu, Y.: The uniqueness of positive solution for a fractional order model of turbulent flow in a porous medium. Appl. Math. Lett. 37, 26-33 (2014)

39. Kong, L.: Second order singular boundary value problems with integral boundary conditions. Nonlinear Anal., Theory Methods Appl. 72, 2628-2638 (2010)

40. Wei, Y., Bai, Z., Sun, S.: On positive solutions for some second-order three-point boundary value problems with convection term. J. Inequal. Appl. 2019, 72 (2019)

41. Jiang, J., Liu, L., Wu, Y.: Second-order nonlinear singular Sturm-Liouville problems with integral boundary problems. Appl. Math. Comput. 215, 1573-1582 (2009)

42. Zhang, X., Liu, L., Wiwatanapataphee, B., Wu, Y.: The eigenvalue for a class of singular $p$-Laplacian fractional differential equations involving the Riemann-Stieltjes integral boundary condition. Appl. Math. Comput. 235, 412-422 (2014)

43. Li, P., Feng, M., Qin, P.: A class of nonlocal indefinite differential systems. Bound. Value Probl. 2018, 81 (2018)

44. Boucherif, A.: Second-order boundary value problems with integral boundary conditions. Nonlinear Anal., Theory Methods Appl. 70, 364-371 (2009)

45. Feng, M.: Existence of symmetric positive solutions for a boundary value problem with integral boundary conditions. Appl. Math. Lett. 24, 1419-1427 (2011)

46. Yan, F., Zuo, F., Hao, X.: Positive solution for a fractional singular boundary value problem with $p$-Laplacian operator. Bound. Value Probl. 2018, 51 (2018)

47. Karakostas, G.L., Tsamatos, P.Ch.: Multiple positive solutions of some Fredholm integral equations arisen from nonlocal boundary-value problems. Electron. J. Differ. Equ. 2002, 30 (2002)

48. Zhang, X., Liu, L., Wu, Y.: The eigenvalue problem for a singular higher fractional differential equation involving fractional derivatives. Appl. Math. Comput. 218, 8526-8536 (2012)

49. Feng, M., Ge, W.: Positive solutions for a class of $m$-point singular boundary value problems. Math. Comput. Model. 46, 375-383 (2007)

50. Jiang, J., Liu, L., Wu, Y.: Positive solutions for $p$-Laplacian fourth-order differential system with integral boundary conditions. Discrete Dyn. Nat. Soc. 2012, Article ID 293734 (2012)

51. Lan, K:: Multiple positive solutions of semilinear differential equations with singularities. J. Lond. Math. Soc. 63 690-704 (2001)

52. Zhang, X., Liu, L., Wu, Y.: Existence results for multiple positive solutions of nonlinear higher order perturbed fractiona differential equations with derivatives. Appl. Math. Comput. 219, 1420-1433 (2012)

53. Guo, L., Liu, L., Wu, Y.: Existence of positive solutions for singular fractional differential equations with infinite-point boundary conditions. Nonlinear Anal., Model. Control 21, 635-650 (2016)

54. Zhang, X., Liu, L., Wu, Y., Lu, Y.: The iterative solutions of nonlinear fractional differential equations. Appl. Math. Comput. 219, 4680-4691 (2013)

55. Zhang, X., Liu, L., Wu, Y., Wiwatanapataphee, B.: Nontrivial solutions for a fractional advection dispersion equation in anomalous diffusion. Appl. Math. Lett. 66, 1-8 (2017)

56. Feng, M., Du, B., Ge, W.: Impulsive boundary value problems with integral boundary conditions and one-dimensional p-Laplacian. Nonlinear Anal., Real World Appl. 70, 3119-3126 (2009) 
57. Li, P., Feng, M.: Denumerably many positive solutions for a $n$-dimensional higher-order singular fractional differential system. Adv. Differ. Equ. 2018, 145 (2018)

58. Ahmad, B., Alsaedi, A.: Existence of approximate solutions of the forced Duffing equation with discontinuous type integral boundary conditions. Nonlinear Anal., Real World Appl. 10, 358-367 (2009)

59. Sun, F., Liu, L., Zhang, X., Wu, Y.: Spectral analysis for a singular differential system with integral boundary conditions. Mediterr. J. Math. 13, 4763-4782 (2016)

60. Liu, L., Sun, F., Zhang, X., Wu, Y.: Bifurcation analysis for a singular differential system with two parameters via to topological degree theory. Nonlinear Anal., Model. Control 22, 31-50 (2017)

61. Zhang, X., Liu, L., Wu, Y.: Multiple positive solutions of a singular fractional differential equation with negatively perturbed term. Math. Comput. Model. 55, 1263-1274 (2012)

62. Zhang, Y:: Positive solutions of singular sublinear Emden-Fowler boundary value problems. J. Math. Anal. Appl. 185, 215-222 (1994)

63. Zhao, Z:: A necessary and sufficient condition for singular nonlinear second-order boundary value problems to have positive solutions. Annal. Math. JCU 13B, 15-24 (1998)

64. Zhang, X., Tian, Y.: Sharp conditions for the existence of positive solutions for a second-order singular impulsive differential equation. Appl. Anal., 1-13 (2017)

65. Yang, F.: Necessary and sufficient conditions for existence of positive solutions to a class of singular second order boundary value problems. Chin. J. Eng. Math. 25, 281-287 (2008)

66. Pouso, R.: Necessary and sufficient conditions for existence and uniqueness of solutions of second-order autonomous differential equations. J. Lond. Math. Soc. 2, 397-414 (2005)

67. Du, X., Zhao, Z:: A necessary and sufficient condition for the existence of positive solutions to singular sublinear three-point boundary value problems. Appl. Math. Comput. 186, 404-413 (2007)

68. Cid, J., Pouso, R., Enguiça, R.: Sharp conditions for the existence of solutions of second-order autonomous differential equations. Mediterr. J. Math. 42, 191-214 (2007)

69. Zhao, J., Wang, L., Ge, W.: Necessary and sufficient conditions for the existence of positive solutions of fourth order multi-point boundary value problems. Nonlinear Anal. 72, 822-835 (2010)

70. Zhang, X., Feng, M.: The existence and asymptotic behavior of boundary blow-up solutions to the $k$-Hessian equation. J. Differ. Equ. 267, 4626-4672 (2019)

71. Zhang, X., Du, Y.: Sharp conditions for the existence of boundary blow-up solutions to the Monge-Ampère equation Calc. Var. Partial Differ. Equ. 57, 30 (2018)

72. Zhang, X., Feng, M.: Boundary blow-up solutions to the $k$-Hessian equation with a weakly superlinear nonlinearity J. Math. Anal. Appl. 464, 456-472 (2018)

73. Feng, M., Zhang, X.: On a $k$-Hessian equation with a weakly superlinear nonlinearity and singular weights. Nonlinear Anal. 190, 111601 (2020)

74. Zhang, X., Feng, M.: Boundary blow-up solutions to the Monge-Ampère equation: sharp conditions and asymptotic behavior. Adv. Nonlinear Anal. 9, 729-744 (2020)

75. Feng, M., Zhang, X.: On a $k$-Hessian equation with a weakly superlinear nonlinearity and singular weights. Nonlinear Anal. 190, 111601 (2020)

76. Guo, D., Lakshmikantham, V:: Nonlinear Problems in Abstract Cones. Academic Press, New York (1988)

77. Papageorgiou, N.S., Rădulescu, V.D., Repovs, D.D.: Nonlinear Analysis-Theory and Methods. Springer Monographs in Mathematics. Springer, Cham (2019)

\section{Submit your manuscript to a SpringerOpen ${ }^{\circ}$ journal and benefit from:}

- Convenient online submission

- Rigorous peer review

- Open access: articles freely available online

- High visibility within the field

- Retaining the copyright to your article

Submit your next manuscript at $\gg$ springeropen.com 